\title{
On the difficulties of discriminating between major and minor hybrid male sterility factors in Drosophila by examining the segregation ratio of sterile and fertile sons in backcrossing experiments
}

\author{
XULIO R. MASIDE \& HORACIO F. NAVEIRA* \\ Departamento de Biología Celular y Molecular, Facultad de Ciencias, Universidad de La Coruña, Campus da \\ Zapateira, La Coruña, Spain
}

\begin{abstract}
The oberservation of segregation ratios of sterile and fertile males in offspring samples from backcrossed hybrid females is, in principle, a valid method to unveil the genetic basis of hybrid male sterility in Drosophila. When the female parent is heterozygous (hybrid) for a sterility factor with major effects, equal proportions of fertile and sterile sons are expected in her offspring. However, intact (not recombined) chromosome segments of considerable length are expected to give segregation ratios that can not be easily differentiated from the $1: 1$ ratio expected from a single factor. When the phenotypic character under analysis can be determined by combinations of minor factors from the donor species spanning a certain chromosome length, very large offspring samples may be needed to test this alternative hypothesis against the null hypothesis of a single major factor. This is particularly the case of hybrid male sterility determinants in Drosophila.
\end{abstract}

Keywords: Drosophila, hybrid males, major genes, polygenes, recombination, sterility.

\section{Introduction}

In a paper recently published in Heredity, Zeng \& Singh (1995) introduced what they claimed to be a general method for identifying major hybrid male sterility genes in Drosophila. Considering the controversy that exists nowadays on the role actually played by such major factors, as compared with minor ones, or polygenes in the determination of hybrid male sterility in Drosophila (Naveira, 1992; Wu \& Palopoli, 1994), the achievement of a method that allows discrimination between both kinds of factors should be welcomed by everyone interested in this matter. Briefly, there are two hypotheses for the genetic basis of hybrid male sterility. One of them postulates that genes responsible for this disturbance have major effects on the phenotype, meaning that each one of them is able to produce sterility by itself when introgressed in the genetic background of the other species (Coyne \& Charles-

\footnotetext{
*Correspondence.
}

worth, 1989; Orr, 1992; Pantazidis et al., 1993; Zeng \& Singh, 1995). The other one, that sterility factors are actually polygenes, whose individual effects on the phenotype are, in principle, undetectable, meaning that the introgression of any single one of them is not able to produce sterility by itself; only when several such factors are cointrogressed in the genetic background of the recipient species is sterility produced (Naveira \& Fontdevila, 1986, 1991a,b; Naveira, 1992; Cabot et al., 1994; Palopoli \& Wu, 1994; Perez \& Wu, 1995). The generalized version of this latter hypothesis maintains that those minor factors are very numerous, dispersed all over the chromosomes, probably different in the $\mathrm{X}$ and the autosomes, and, most important, are able to interact among themselves to bring about hybrid sterility even when distantly positioned on the chromosome (Naveira \& Fontdevila, 1991a; Naveira, 1992; Palopoli \& Wu, 1994). One of the main observations supporting this model is the apparent existence of a threshold in the chromosome length that must be introgressed to produce male sterility: small 
introgressions do allow fertility, but when those very same small chromosome segments are introgressed together, the resulting hybrid male is sterile (Naveira \& Fontdevila, 1991a,b; Naveira, 1992). It should be clear, then, the importance that the transmission of intact chromosome segments during backcrossing has under this polygenic model. The theoretical distribution of lengths of intact chromosome segments (with reference to the original parental chromosome) has already been worked out (Naveira \& Barbadilla, 1992), and allows one to predict the expected frequencies of individuals bearing introgressed segments with any arbitrary size after any number of generations of backcrossing to a recipient species. In this paper we show that the method introduced by Zeng \& Singh (1995) is generally not appropriate to identify major hybrid male sterility genes, unless the sample size, or the threshold size for introgressions leading to sterility, postulated by the rivalling polygenic hypothesis, are relatively large. That method relies on the production of fertile and sterile sons in roughly equal proportions after backcrossing hybrid females. However, intact chromosome segments of considerable length may give segregation ratios not statistically different from those of a single genetic factor. Only with very large samples is it possible to discriminate between both alternatives when the intact chromosome segment is only a few map units long. In particular, in the case of the hybrids between Drosophila simulans and D. mauritiana presented by Zeng \& Singh to illustrate their method, their results can be shown to be perfectly compatible with the expectations of the polygenic model, if hybrid male sterility is actually being produced by the introgression of at least 5-10 real map units of the $\mathrm{X}$ chromosome from mauritiana into simulans, an assumption that is in perfectly good agreement with former results in this respect with these same hybrids (Naveira, 1992).

\section{Results}

Perhaps the best measure we can have of true genetic distance along a chromosome region is $m$, the average number of crossovers per meiosis in that region. The proportion of meiosis with at least one crossover is one minus the fraction with zero crossovers which, assuming no interference, may be derived from a Poisson distribution of parameter $m$. Only one-half of the products of those meioses will be recombinants in the region of interest. Hence $c=\left(1-e^{-m}\right) / 2$. As shown by Haldane (1919), this relationship, usually denoted the mapping function, is approximately linear, namely $c \sim m / 2$, for a certain range corresponding to very small $m$ values (genetic distances). Thus, using this linear relationship, the number of real map units of any chromosome region can be obtained ( 1 real map unit $=0.02$ crossovers per meiosis per chromosome, i.e. real map units $=50 \mathrm{~m}$; Suzuki et al., 1986, p. 124).

Consider an $F_{1}$ hybrid between two species. This hybrid will be backcrossed to one of the parental species, which we denote as the recipient species, the other one being the donor species. In meiosis in this hybrid, each chromosome is present as two homologues, each one from a different species, and each one consisting of two sister chromatids. Let the length of an arbitrarily chosen chromosome region be $m$ in the scale of crossovers per meiosis $(50 \mathrm{~m}$ map units in the real map scale), i.e. an average of $m$ crossovers would take place in that chromosome interval per meiosis. If there is not interference, the probability of no crossover in this interval in one generation is given by the Poisson distribution, namely $e^{-m}$. Of the four products of this meiosis, only two of them will receive the chromosome interval from the donor parental species (the other two products will receive the homologous chromosome interval from the recipient species). Then, the probability of a gamete receiving an intact chromosome interval would be $e^{-m} / 2$, considering only the contribution from meioses with no crossovers in that interval. By the same rationale, it can be shown that the probability that only one crossover takes place in the region considered, and a resulting gamete receives an intact interval, is given by $m e^{-m} / 4$. This is enough for our purposes, because the contribution from meioses with two or more crossovers to the gametic pool with intact segments is comparatively much smaller. We may conclude, then, that the probability that a gamete from the $F_{1}$ female receives an intact chromosome interval of $50 \mathrm{~m}$ real map units from the donor species is:

$p=e^{-m} / 2+m e^{-m} / 4$.

Table 1 shows these probabilities for a range of chromosome lengths, from 1 to 70 real map units, with its equivalence in centimorgans (cM). Even for the largest of these sizes, which corresponds approximately to the full length of chromosome $\mathrm{X}$ in $D$. melanogaster, a considerable frequency of gametes (21 per cent) is expected to receive the intact chromosome from the donor species. If this is so, it can be anticipated that the discrimination between the segregation ratios for a single heterozygous locus (1:1) and an intact chromosome segment $(p: 1-p)$ will not always be an easy task. The problem, a 
Table 1 Probability $(P)$ that a gamete receives the intact (not recombined) chromosome segment introgressed in a hybrid heterozygous female, for different sizes (in real map units) of the involved segments. Probability is calculated from eqn 1 in the text

\begin{tabular}{lccc}
\hline $\begin{array}{l}\text { Length of } \\
\text { introgressed } \\
\text { chromosome } \\
\text { segment in } \\
\text { the female } \\
\text { (map units) }\end{array}$ & $\begin{array}{c}\text { Average number } \\
\text { of crossovers } \\
\text { per meiosis } \\
\text { in that region } \\
(m)\end{array}$ & $\begin{array}{c}\text { Equivalence } \\
\text { in } \\
\text { centimorgans } \\
(\mathrm{cM})\end{array}$ & $\begin{array}{c}\text { Probability } \\
\text { that a gamete } \\
\text { receives the } \\
\text { segment intact } \\
(p)\end{array}$ \\
\hline 1 & 0.02 & 0.99 & 0.495 \\
5 & 0.10 & 4.76 & 0.475 \\
10 & 0.20 & 9.06 & 0.450 \\
15 & 0.30 & 12.96 & 0.426 \\
20 & 0.40 & 16.48 & 0.402 \\
25 & 0.50 & 19.67 & 0.379 \\
30 & 0.60 & 22.56 & 0.357 \\
35 & 0.70 & 25.17 & 0.335 \\
40 & 0.80 & 27.53 & 0.315 \\
50 & 1.00 & 31.61 & 0.276 \\
70 & 1.40 & 37.67 & 0.210 \\
\hline
\end{tabular}

simple one in hypothesis testing (Sokal \& Rohlf, 1981, section 7.8), is illustrated in Fig. 1. It is our hypothesis that the $F_{1}$ female is heterozygous for a Mendelian factor, whose segregation determines with complete penetrance an easily observable phenotypic character in the offspring. Let us call this hypothesis $H_{0}$, the null hypothesis. It means that, on average, half the offspring of the hybrid heterozygous female will receive the donor species allele ( $\mu_{0}=0.5 n$, where $n$ designates the total sample size analysed in the offspring), and thus exhibit the corresponding diagnostic phenotype, If $n$ is sufficienty large $(\geq 30)$, the binomial distribution, with $p=q=0.5$, is approximately $N\left(0.5 n, 0.5 n^{0.5}\right)$, as presented in Fig. 1. Our alternative hypothesis $\left(H_{1}\right)$ states that the phenotypic character under analysis (hybrid male sterility, in this case) is determined not by a single gene, but by a combination of genes spanning a chromosome segment of $50 \mathrm{~m}$ map units. Again, the corresponding, binomial distribution is approximately $N\left(n p,(n p q)^{0.5}\right)$, where $p$ is given by (1), and $q=1-p$. The power of the test, i.e. the probability of rejecting the null hypothesis when in fact it is false and the alternative hypothesis is correct, corresponds to the area $1-\beta$ in Fig. 1, where $\beta$ designates the probability of type II errors. Obviously, we would like to have $1-\beta$ as large as possible, but it falls off sharply as the alternative hypothesis approaches the null hypothesis, as we have tried to illustrate in Fig. 1. Everyone probably

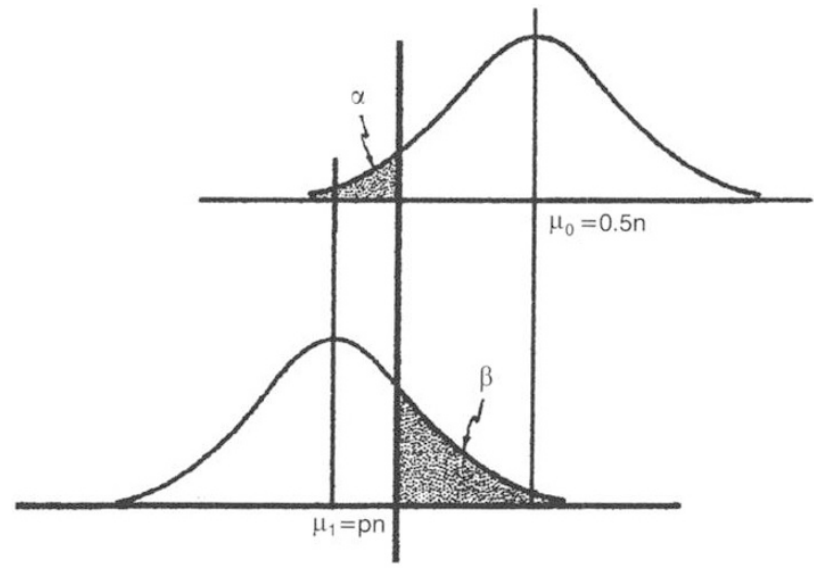

Fig. 1 Normal approximations, for large offspring sample sizes $(n>30)$ of sampling distributions from experiments with either an expected percentage of 50 per cent sterile and 50 per cent fertile sons (upper curve, null hypothesis), or less than 50 per cent steriles (lower curve, alternative hypothesis). $\mu$ : average number of sterile sons in samples of size $n . \alpha$ : probability of type I errors. $\beta$ : probability of type II errors.

knows that to improve the power of a given test (decrease $\beta$ ) while keeping the probability of type I errors $(\alpha$; level of significance $=100 \alpha$ per cent $)$ constant for a stated null hypothesis, the sample size must be increased. In Table 2 , we show the offspring sample sizes that should be used to reduce the prob- 
Table 2 Offspring sample sizes that should be used to discriminate the segregation of a single genetic factor from that of an intact chromosome segment in heterozygosis in a female parent

\begin{tabular}{lrr}
\hline & \multicolumn{2}{c}{$\begin{array}{c}\text { Sample size to make } \\
\text { the probability of type } \\
\text { II errors under } H_{0}\end{array}$} \\
$\begin{array}{l}\text { Length of introgressed } \\
\text { chromosome segment } \\
\text { in map units }\end{array}$ & \multicolumn{2}{c}{$(\alpha=0.05)$ become } \\
\cline { 2 - 3 } 1 & 0.05 & 0.01 \\
\hline 5 & 108894 & 158379 \\
10 & 4350 & 6326 \\
15 & 1083 & 1575 \\
20 & 492 & 714 \\
25 & 278 & 403 \\
30 & 180 & 261 \\
35 & 128 & 184 \\
40 & 94 & 136 \\
50 & 74 & 106 \\
70 & 49 & 69 \\
& 27 & 37 \\
\hline
\end{tabular}

The null hypothesis ( $H_{0}$, a single genetic factor) predicts equal proportions of two phenotypic classes. Several alternatives are considered, which postulate that the character under analysis is determined by a combination of factors spanning a chromosome segment of the indicated length. A fixed significance level of 5 per cent is adopted for the test. Sample sizes for the power of the test to become either 0.95 , or 0.99 are indicated.

abilities of type II errors down to the usual values of 0.05 and 0.01 , keeping $\alpha=0.05$, when testing alternative hypotheses differing in the length of the introgressed chromosome interval considered in the hybrid mother. It can be seen that, as expected, the segregation of very small chromosome regions (1 map unit) can not be distinguished, in practice, from that of a single gene in heterozygosis, as we should have to examine tens of thousands of individuals. The situation improves considerably for regions with 5-10 map units, but still we need samples of the order of thousands individuals. Only when the introgressed segment is relatively long (at least 15 map units), is hypothesis testing feasible with a modest sample size. Interestingly, we found that even for very long chromosome segments, such as 70 map units (the whole length of the $\mathrm{X}$ chromosome in $D$. melanogaster), we should examine at least 27 individuals in the offspring of the hybrid female to be reasonably confident $(\beta=0.05)$ that the character we are examining is not determined by the whole chromosome block received from the donor species.

Up to now, we have been considering the results of backcrossing an $F_{1}$ hybrid female. When backcrosses are repeated for several generations, the average length of intact chromosome segments (with reference to the original parental chromosome) or, equivalently, the average length of segments which are still heterozygous (with reference to the fully heterozygous $F_{1}$ chromosome), may diminish, but cannot increase. Exact equations both for the average and standard deviation of intact lengths, after any number of generations of backcrossing, have been derived elsewhere (Naveira \& Barbadilla, 1992). The expected intact chromosome length decreases very quickly up to the fourth generation (for example, the $\mathrm{X}$ chromosome in $D$. melanogaster would be reduced to 40 map units, on average), and then more and more slowly. After ten generations, the average intact size is 20 map units, 10 map units after 20 generations, and still 5 map units after 40 generations. This means that all the results derived in this paper for segments of up to 10 map units long, for the first backcross of an $F_{1}$ hybrid female, will be in practice applicable to females obtained after a considerable number of generations of backcrossing. And this rather long intact chromosome segments are expected to be still segregating during at least the first ten generations of a backcrossing experiment.

Entering now into the details of the application of the method introduced by Zeng \& Singh (1995) to the hybrids between $D$. simulans and $D$. mauritiana, the first thing that should be pointed out is the small size of the offspring samples analysed by these authors (only 16 males, on average). This means that, at least for the first ten backcrosses they report, their results are not useful to decide whether a single major sterility factor, or either a chromosome segment, is bringing about the sterility they observe. A second consideration is that to pool data from different backcross generations, as they do in their Table 3 , is a highly questionable procedure if the alternative hypothesis to be tested is in any way connected with the size of the introgressed segments, because the distribution of the sizes of these segments changes from one generation to the next. All these comments should be understood not as an attempt to reduce the merits of Zeng \& Singh's paper, but to illustrate the difficulties of testing adequately the different hypotheses that may explain the observed data. However, even if all these objections are disregarded, the pooled data offered by Zeng \& Sing ( 370 fertile vs. 348 sterile males) do not differ significantly from those expected under the polygenic hypothesis, if the threshold size of the introgressed segments to produce sterility is 10 map 
Table 3 Segregation ratio of fertile and sterile sons observed in backcross generations by Zeng \& Singh (1995), and expected (in a single generation) if sterility is determined by the introgression of combinations of factors spanning a chromosome segment of arbitrary size (threshold size for sterility)

\begin{tabular}{|c|c|c|c|c|c|}
\hline \multirow{2}{*}{$\begin{array}{l}\text { Offspring } \\
\text { males }\end{array}$} & \multirow{2}{*}{$\begin{array}{l}\text { No. observed } \\
\text { by } \\
\text { Zeng \& Singh }\end{array}$} & \multicolumn{4}{|c|}{$\begin{array}{l}\text { No. expected if the introgressed } \\
\text { segment, corresponding to the threshold } \\
\text { for sterility, has the indicated length } \\
\text { (map units) }\end{array}$} \\
\hline & & 1 & 5 & 10 & 15 \\
\hline \multirow{4}{*}{$\begin{array}{l}\text { Sterile } \\
\text { Fertile }\end{array}$} & 348 & 355 & 341 & 323 & 306 \\
\hline & 370 & 363 & 377 & 395 & 412 \\
\hline & & 0.137 & 0.137 & 1.748 & 4.953 \\
\hline & bility & 0.712 & 0.712 & 0.186 & 0.026 \\
\hline
\end{tabular}

units or less (Table 3). By threshold size we mean that chromosome segments from the donor species (D. mauritiana) whose length exceeds the threshold, give rise to sterile males when recombined in the genome of the recipient species (D. simulans); those segments whose length is smaller than the threshold, should allow fertility of the hybrids. The higher the threshold, the lower the probability of a son receiving an intact chromosome segment at least that long from the hybrid mother. An additional comment on this respect is pertinent: to exclude the offspring data from females which produced only one or two sterile males, as Zeng \& Singh do, may be acceptable when the number of sons is high, but in no way when the observed numbers per family range from seven to 17 . If the data discarded by Zeng \& Singh from their Table 2 are taken into consideration (from $\mathrm{BC}_{4}$ onward), a total of 459 fertile and 360 sterile males are obtained. These numbers are compatible even with the hypothesis of sterility being produced by the introgression of a chromosome segment of 20 map units $\left(\chi_{1}^{2}=2.376\right.$, $P=0.124$ ), which corresponds to nearly 30 per cent of the full length of the $\mathrm{X}$ chromosome. It must be also clearly understood that, if the polygenic hypothesis is correct, the method applied by Zeng \& Singh should lead to the selection as progenitors, one generation after the other, of those families that still keep an intact chromosome segment from the one donor species whose size is above the threshold for sterility. Therefore, the argument that the segregation ratio has been observed for quite a large number of backcross generations (namely 45 in the experiment reported by Zeng \& Singh) is irrelevant, except to state that, after so many generations, if there is no selection on hybrid viability, the size of the intact chromosome segment still introgressed from the donor species will most probably be very near the threshold size for hybrid sterility.

A final argument militates against the null hypothesis of a single Mendelian factor, and in favour of the alternative polygenic model. After the application of their method to the mauritiana/simulans hybrids, Zeng \& Singh conclude that they have introgressed a single Mendelian factor of hybrid male sterility $(\mathrm{hms})$ from mauritiana into simulans. They mapped such a putative single factor to the $\mathrm{X}$ chromosome, quite near $(6 \mathrm{cM})$ the forked locus. Their results in this respect, given in their Table 6, indicate an extreme asymmetry in the frequencies of the two kinds of recombinant males expected after their mapping crosses (five fertile wild-type males vs. 63 sterile mutants). This is precisely what is expected under the polygenic model, which predicts a difference in the 'apparent' genetic distance to a putative sterility locus (which in fact does not exist) calculated from each of the two alleles of the marker (Maside \& Naveira, 1996), this difference consisting in an excess of the sterile mutant recombinant class, that would be most conspicuous when the threshold size for sterility is small, in terms of the total length of the introgressed chromosome segment.

\section{Discussion and conclusions}

The method introduced by Zeng \& Singh (1995) for detecting major hybrid male sterility genes, based on the selection of backcross females which produce fertile and sterile sons in approximately equal proportions, is valid in principle. However, extreme caution should be exercised to increase the power of 
the test of the null hypothesis (a single major gene) against the alternatives (an intact chromosome segment). The first ten generations of the backcrossing protocol are useless for testing these hypotheses, because both the expected average and standard deviation of intact chromosome sizes are too high (about 20 and 10 map units, respectively according to Naveira \& Barbadilla, 1992, Figs 1 and 2). The backcrossing protocol should be continued for at least 20 generations to approach limiting functions for those two parameters. From then on, family data from different generations can be pooled to investigate the segregation ratio. However, the total numbers to be used must be very high if the null hypothesis is going to be tested against an alternative based on small threshold sizes for hybrid sterility. In the case of the hybrids between $D$. mauritiana and $D$. simulans, the results of the experiments that produce sterile males by recombining fertile introgressions associated to the loci yellow, white and forked (Naveira, 1992) indicate that the threshold size is not higher than 10 map units. The segregation of an intact introgressed chromosome segment of that size would be rather difficult to differentiate from that of a single major factor of hybrid male sterility: at least 1083 sons should be necessary for the power of the test to become 0.95 , with a significance level of 5 per cent. If the results from the chromosome regions associated to yellow, white and forked are representative of the distribution of thresholds along the whole $\mathrm{X}$ chromosome, then it is clear that it would be nearly impossible to detect major hybrid male sterility factors in the pair mauritiana-simulans (and probably in the pair sechelliasimulans, also) by the analysis of segregation ratios of fertile and sterile sons

\section{Acknowledgements}

This work was supported by grant PB92-0386 awarded to $\mathrm{HN}$ by the DGICYT, Ministerio de Educación y Ciencia, and a research sholarship awarded to X. M. by the Xunta de Galicia, Spain.

\section{References}

CABOT, E. L., DAVIS, A. W., JOHNSON, N. A. AND WU, C.-I. 1994. Genetics of reproductive isolation in the Drosophila simulans clade: complex epistasis underlying hybrid male sterility. Genetics, 137, 175-189.

COYNE, J. A. AND CHARLESWORTH, B. 1989. Genetic analysis of X-linked sterility in hybrids between three sibling species of Drosophila. Heredity, 62, 97-106.

HALDANE, J. B. S. 1919. The combination of linkage values, and the calculation of distances between the loci of linked factors. J. Genet., 8, 299-309.

MASIDE, X. R. AND NAVEIRA, H. F. 1996. A polygenic basis of hybrid sterility may give rise to spurions localizations of major sterility factors. Heredity, 77 (in press).

NAVEIRA, H. F. 1992. Location of X-linked polygenic effects causing sterility in male hybrids of Drosophila simulans and D. mauritiana. Heredity, 68, 211-217.

NAVEIRA, H. F. AND BARBADILLA, A. 1992. The theoretical distribution of lengths of intact chromosome segments around a locus held heterozygous with backcrossing in a diploid species. Genetics, 130, 205-209.

NAVEIRA, H. F. AND FONTDEVILA, A. 1986. The evolutionary history of Drosophila buzzatii. XII. The genetic basis of sterility in hybrids between $D$. buzzatii and its sibling $D$. serido from Argentina. Genetics, 144, 841-857.

NAVEIRA, H. F. AND FONTDEVILA, A. 1991a. The evolution history of Drosophila buzzatii. XXII. Chromosomal and genic sterility in male hybrids of $D$. buzzatii and $D$. koepfereae. Heredity, 66, 233-239.

NAVE1RA, H. F. AND FOnTDEVILA, A. 1991b. The evolutionary history of Drosophila buzzatii. XXI. Cumulative action of multiple sterility factors on spermatogenesis in hybrids of D. buzzatii and D. koepferae. Heredity, 67, $57-72$.

ORR, H. A. 1992. Mapping and characterization of a 'speciation gene' in Drosophila. Genet. Res., 59, 73-80.

PALOPOLI, M. F. AND WU, C.-1. 1994. Genetics of hybrid male sterility between Drosophila sibling species: a complex web of epistasis is revealed in interspecific studies. Genetics, 138, 329-341.

PEREZ, D. E. AND WU, C.-I. 1995. Further characterization of the Odysseus locus of hybrid sterility in Drosophila: one gene is not enough. Genetics, 140, 201-206.

PANTAZIDIS, A. C., GALANOPOUlos, V. K. AND Zouros, E. 1993. An autosomal factor from Drosophila arizonae restores normal spermatogenesis in D. mojavensis males carrying the arizonae $\mathrm{Y}$ chromosome. Genetics, $\mathbf{1 3 4}$, 309-318.

SOKAL, R. R. AND ROHLF, F. J. 1981. Biometry, 2nd edn. Freeman, New York.

SUZUKI, D. T., GRIFFITHS, A. J. F., MILLER, J. H. AND LEWONTIN, R. C. 1986. An Introduction to Genetic Analysis, 4th edn. Freeman, New York.

WU, C.-I. AND PALOPOLI, M. F. 1994. Genetics of postmating reproductive isolation in animals. Ann. Rev. Genet., 28, 283-308.

ZENG, L.-W. AND SINGH, R. S. 1995. A general method for identifying major hybrid male sterility genes in Drosophila. Heredity, 75, 331-341. 